\title{
EDITORIAL
}

\section{Atrial fibrillation ablation in the real world}

\author{
F R Quinn, A C Rankin
}

Heart 2005;91:1507-1508. doi: 10.1136/hrt.2004.059212

\section{While the potential for curative treatment of atrial fibrillation has generated much interest in the role of catheter ablation, its widespread use has been limited by a number of factors}

$\mathrm{T}$ he observation in the late 1990s that ectopic beats originating from the pulmonary veins could act as triggers for the initiation of atrial fibrillation (AF) opened up the possibility of curative treatment by catheter ablation. ${ }^{1}$ The potential for successful treatment has been confirmed, ${ }^{2-4}$ but the applicability of such complex and invasive procedures to the large number of patients with AF remains uncertain. What is the role of catheter ablation for AF in the "real world"?

\section{EVOLUTION OF TECHNIQUE}

The optimal method of catheter ablation for AF has been hotly debated. The initial technique was to identify and ablate the "culprit" focus within the pulmonary vein, ${ }^{2}$ but this approach was limited by the complication of pulmonary vein stenosis and the recognition that multiple veins were involved in the majority of patients. ${ }^{14}$ The technique evolved to aim for electrical isolation of the pulmonary veins, which can be achieved by segmental ablation guided by potentials from a multipole catheter positioned within the ostium of the vein. ${ }^{5}$ Around the same time, an alternative approach was developed, involving more extensive ablation of the left atrium, with wider circumferential lesions around the pulmonary veins, guided by sophisticated three dimensional electroanatomical mapping. ${ }^{3}$ This method alters not only the triggers, but also the substrate for the arrhythmia, and can include additional linear atrial lesions. The initial report of this technique suggested higher success rates than the electrophysiologically guided segmental pulmonary vein isolation. ${ }^{3}$ A randomised trial comparing the two methodologies in paroxysmal AF confirmed this improved outcome, with $88 \%$ of patients who underwent circumferential ablation free of AF at six months, compared to $67 \%$ of those who had segmental isolation. ${ }^{6}$ Similar findings were reported in a non-randomised study of consecutive patients, with $75 \%$ and $60 \%$ of patients free from AF following circumferential and segmental ablation, respectively. ${ }^{7}$

Correspondence to: Dr Andrew C Rankin, Department of Medical Cardiology, Glasgow Royal Infirmary, 10 Alexandra Parade, Glasgow G31 2ER, UK; acrla@clinmed.gla.ac.uk

\section{LIMITATIONS OF CATHETER ABLATION FOR AF}

The potential for curative treatment of the most common cardiac arrhythmia, with its association with significant morbidity and mortality, has generated much interest in catheter ablation for AF. However, a number of factors have limited its widespread use. Initial enthusiasm was tempered by long procedure times and limited success rates, often with the need for repeat procedures. In addition, it became evident that the technique carried the threat of potentially life threatening complications, such as cardiac tamponade and embolic stroke. Success rates have improved with the evolution and development of techniques, but more extensive ablation has brought the spectre of new complications, such as atrio-oesophageal fistulae. ${ }^{8}$ The majority of studies report higher success rates in patients with paroxysmal, compared to persistent, AF. Technology in the field is rapidly advancing, and complication rates may be improved further by having better three dimensional anatomical information-for example, derived from computed tomography or magnetic resonance imaging-and alternative ablation energy sources such as focused ultrasound. ${ }^{9}$

\section{AF ABLATION IN THE "REAL WORLD"}

A recent worldwide survey reported 8745 patients treated at 181 centres. ${ }^{10}$ The numbers per year increased from 18 patients in 1995 to 5050 in 2002. The majority underwent segmental pulmonary vein isolation, $27.3 \%$ had more than one procedure, and major complications occurred in $6.0 \% ; 52 \%$ became asymptomatic, and a further $23.9 \%$ were improved by antiarrhythmic drugs. Also of note is the fact that centres which had performed the most procedures tended to have the highest success rates.

Pioneering institutions worldwide have now performed ablation for AF on thousands of patients, but can their results be reproduced in lower volume centres? The short report in this issue of Heart by Pontoppidan et al describes the experience of one such electrophysiology centre. ${ }^{11}$ This is a non-randomised observational retrospective study, with all the attendant limitations, but is of interest as their experience reflects the progression of the technique elsewhere. Their initial success rate with segmental pulmonary vein isolation was only $25 \%$, with a further $25 \%$ improved on drugs. The rate of freedom from $\mathrm{AF}$ rose to $73 \%$ when they changed to circumferential ablation, with a reduction in the number of repeat procedures. Cardiac tamponade occurred in $4 \%$ with both methods, and one patient had a transient neurological deficit. Another recent report in Heart from a "nonpioneering" centre reported 55\% success at six month follow up following a first procedure, in a consecutive series of 100 patients. ${ }^{12}$ Cardiac tamponade requiring pericardiocentesis occurred in six patients, right coronary artery embolus in 
two, and complete heart block in one. Again, the technique evolved during the study, from targeting only arrhythmogenic veins, to attempting to isolate all four pulmonary veins, and the addition of linear lesions in the atrium. These two studies have demonstrated that the technique of pulmonary vein isolation for $\mathrm{AF}$ can be introduced into experienced electrophysiology centres with promising results and a complication rate comparable to that reported in the wider literature.

\section{"RATE VERSUS RHYTHM"}

The benefits of maintaining sinus rhythm have been questioned by the "rate versus rhythm" debate, with evidence of possible harm from a pharmacologically based, rhythm control strategy. ${ }^{13}$ However, there is growing evidence that the maintenance of sinus rhythm following catheter ablation for AF can provide clinical and prognostic benefits. A non-randomised study examining outcome in 589 patients following catheter ablation for AF showed improved mortality, morbidity, and quality of life, compared to 582 medically treated patients. ${ }^{14}$ Indeed, the overall survival of ablated patients was no different to that of the general population, matched for age and sex. Additionally, a study of ablation in patients with congestive heart failure and atrial fibrillation, the majority of whom were in persistent $\mathrm{AF}$, reported improvements in cardiac function, symptoms and quality of life. ${ }^{15}$

\section{CONCLUSIONS}

Curative ablative treatment for AF is a clinical reality, but has its limitations, including failure rates of around 25\%, recurrences with the need for repeat procedures, and the potential for serious complications. It demands high levels of expertise, including the transseptal puncture technique and skills in catheter manipulation in the left atrium. There is a requirement for trained support staff, and the procedure is facilitated by expensive modern technology. These considerations will restrict its use to specialist electrophysiology centres. As such, the technique is not currently applicable to the majority of patients with AF, many of whom are frail and elderly. However, it is now appropriate that catheter ablation for AF should be available to treat, and possibly cure, those many patients with troublesome AF in whom medical treatment has failed. This will require the expansion of electrophysiology services in many parts of the world. As techniques continue to evolve, and the understanding of this complex arrhythmia advances, the procedural success and safety may improve making it available to more of our patients.

\section{Authors' affiliations}

F R Quinn, A C Rankin, Department of Medical Cardiology, Glasgow Royal Infirmary, Glasgow, UK

Competing interests: None.

\section{REFERENCES}

1 Haissaguerre $M$, Jais $P$, Shah DC, et al. Spontaneous initiation of atrial fibrillation by ectopic beats originating in the pulmonary veins. N Engl J Med 1998;339:659-66

2 Haissaguerre $M$, Jais $P$, Shah DC, et al. Catheter ablation of chronic atrial fibrillation targeting the reinitiating triggers. J Cardiovasc Electrophysiol 2000;11:2-10.

3 Pappone C, Rosanio S, Oreto G, et al. Circumferential radiofrequency ablation of pulmonary vein ostia: a new anatomic approach for curing atrial fibrillation. Circulation 2000;102:2619-28.

4 Haissaguerre $M$, Jais $P$, Shah DC, et al. Electrophysiological end point for catheter ablation of atrial fibrillation initiated from multiple pulmonary venous foci. Circulation 2000;101:1409-17.

5 Haissaguerre $M$, Shah DC, Jais $P$, et al. Electrophysiological breakthroughs from the left atrium to the pulmonary veins. Circulation 2000;102:2463-5.

6 Oral H, Scharf C, Chugh A, et al. Catheter ablation for paroxysmal atrial fibrillation: segmental pulmonary vein ostial ablation versus left atrial ablation. Circulation 2003;108:2355-60.

7 Mansour M, Ruskin J, Keane D. Efficacy and safety of segmental ostial versus circumferential extra-ostial pulmonary vein isolation for atrial fibrillation. J Cardiovasc Electrophysio 2004;15:532-7.

8 Pappone C, Oral H, Santinelli V, et al. Atrio-esophageal fistula as a complication of percutaneous transcatheter ablation of atrial fibrillation. Circulation 2004; 109:2724-6.

9 Rosanio S, Ware DL, Saeed M. Pulmonary vein ablation of atrial fibrillation: beyond the traditional. Am J Med Sci 2004;328:323-9.

10 Cappato R, Calkins H, Chen S-A, et al. Worldwide survey on the methods, efficacy, and safety of catheter ablation for human atrial fibrillation. Circulation 2005; 111:1100-5.

11 Pontoppidan J, Nielsen JC, Poulsen SH, et al. Radiofrequency ablation of atrial fibrillation: effectiveness and safety in 102 consecutive patients. Heart 2005;91:1611-12

12 Bourke JP, Dunuwille A, O'Donnell D, et al. Pulmonary vein ablation for idiopathic atrial fibrillation: six month outcome of first procedure in 100 consecutive patients. Heart 2005;91:51-7.

13 Corley SD, Epstein AE, DiMarco JP, et al. Relationships between sinus rhythm, treatment, and survival in the atrial fibrillation follow-up investigation of rhythm management (AFFIRM) study. Circulation 2004; 109:1509-13.

14 Pappone C, Rosanio S, Augello G, et al. Mortality, morbidity, and quality of life after circumferential pulmonary vein ablation for atrial fibrillation: outcomes from a controlled nonrandomized long-term study. J Am Coll Cardiol 2003;42:185-97

15 Hsu LF, Jais $P$, Sanders $P$, et al. Catheter ablation for atrial fibrillation in congestive heart failure. N Engl J Med 2004;351:2373-83.

\section{bmjupdates+}

bmjupdates+ is a unique and free alerting service, designed to keep you up to date with the medical literature that is truly important to your practice.

bmjupdates+ will alert you to important new research and will provide you with the best new evidence concerning important advances in health care, tailored to your medical interests and time demands.

Where does the information come from?

bmjupdates+ applies an expert critical appraisal filter to over 100 top medical journals A panel of over 2000 physicians find the few 'must read' studies for each area of clinical interest

Sign up to receive your tailored email alerts, searching access and more...

www.bmjupdates.com 